\begin{tabular}{|c|l|}
\hline Title & Persistent currents in Möbius strips \\
\hline Author(s) & Y akubo, Kousuke; A vishai, Y shai; Cohen, Doron \\
\hline Citation & $\begin{array}{l}\text { PHY SICAL REVIEW B, 67, 125319 } \\
\text { https://doi.org/10.1103/PhysRevB.67.125319 }\end{array}$ \\
\hline Issue Date & 2003-03-31 \\
\hline Doc URL & http://hdl.handle.net/2115/5649 \\
\hline Rights & Copyright $\odot 2003$ A merican Physical Society \\
\hline Type & article \\
\hline File Information & PRB67.pdf \\
\hline
\end{tabular}

Instructions for use 


\title{
Persistent currents in Möbius strips
}

\author{
Kousuke Yakubo, ${ }^{1}$ Yshai Avishai, ${ }^{1,2,3}$ and Doron Cohen $^{2}$ \\ ${ }^{1}$ Department of Applied Physics, Hokkaido University, Sapporo 060-8628, Japan \\ ${ }^{2}$ Department of Physics, Ben Gurion University, Beer Sheva, Israel \\ ${ }^{3}$ Ilse Katz Center for Nanotechnology, Ben Gurion University, Beer Sheva, Israel
}

(Received 2 January 2003; published 31 March 2003)

\begin{abstract}
The relation between the geometry of a two-dimensional sample and its equilibrium physical properties is exemplified here for a system of noninteracting electrons on a Möbius strip. Dispersion relation for a clean sample is derived and its persistent current under moderate disorder is elucidated, using statistical analysis pertinent to a single sample experiment. The flux periodicity is found to be distinct from that in a cylindrical sample, and the essential role of disorder in the ability to experimentally identify a Möbius strip is pointed out.

DOI: 10.1103/PhysRevB.67.125319

PACS number(s): 73.23.Ra, 72.15.Rn, 73.23.Ad
\end{abstract}

\section{INTRODUCTION}

An important theme in quantum mechanics is to find a relation between a global geometry of a sample (e.g., boundary conditions) and its physical properties. We address this issue by comparing flux periodicity of persistent currents in a cylinder and in a Möbius strip. The aim is to determine whether the geometrical (in some sense topological) difference is tangible and experimentally observable. At zero temperature, the persistent current $I(\phi)$ in a ring can be expressed as ${ }^{1}$

$$
I(\phi)=-\frac{\partial E(\phi)}{\partial \phi}=\sum_{n=1}^{\infty} I_{n} \sin (2 \pi n \phi),
$$

where $\phi$ is the magnetic flux threading the ring in units of $\Phi_{0}=h c / e, E(\phi)$ is the ground-state energy, and $I_{n}$ are the current harmonics.

The current $I(\phi)$ is an antisymmetric and periodic function of $\phi$ with period 1 . Possible occurrence of smaller flux periodicity in mesoscopic physics is one of the cornerstones of weak localization. For the cylinder geometry, conductance measurements ${ }^{2}$ and magnetization of $10^{7}$ copper rings ${ }^{3}$ indicate the emergence of periodicity $1 / 2$. It is shown to be intimately related to the procedure of averaging over disorder realizations and numbers of electrons in the rings. ${ }^{4-6,2}$ Very recently, a microscopic $\mathrm{NbSe}_{3}$ Möbius strip has been fabricated. ${ }^{7}$ Obviously, in this case, attention should be focused on a single sample measurement ${ }^{8}$ for which there is no self-averaging.

Let us first mention several intuitive points relevant to the flux periodicity in the Möbius strip, based on semiclassical arguments and geometry. ${ }^{9}$ First, recall that the periodicity is related to interference between trajectories (such as Aharonov-Bohm interference between different trajectories or weak-localization interference between time-reversed paths). In the cylinder (Möbius) geometry, an electron moving in the longitudinal direction along the ring encircles the system once (twice) before returning to its initial position. Therefore, we might expect different flux periodicities of the persistent current between the two cases. Second, unlike a cylinder which can be "pressed" into a one-dimensional ring, the Möbius strip cannot be pressed into a one- dimensional structure. This brings in another important factor, namely, the motion of electrons in the transverse direction. In a tight-binding model this motion is controlled by the transverse hopping. If it is very weak, the twice-encircling property of the Möbius strip implies the dominance of even harmonics $I_{2 n}$. On the contrary, for a strong transverse hopping, the current in the Möbius strip is expected to be effectively similar to that in the cylindrical strip. ${ }^{10}$ In the following we are mainly interested in a regime where the transverse hopping is slightly less than longitudinal one. Third, the role of disorder should be carefully examined. Weak disorder is not expected to significantly alter interference between semiclassical trajectories discussed above, while strong disorder should result in a reduced sensitivity to the pertinent geometry, due to localization effects. The most intriguing disorder effect might then be expected in a moderate strength of disorder which will be used below. The upshot of the present study is that the periodicity pattern in a Möbius strip is remarkably distinct from that of a cylinder, and that disorder plays a crucial role in making the statistical effect detectable.

\section{MODEL}

A Möbius strip is modeled by considering a noninteracting particle in a rectangle of length $L_{x}$ and width $L_{y}$, requiring its wave function $\psi(x, y)$ to satisfy Dirichlet boundary conditions $(\mathrm{BC})$ in the $y$ direction, and Möbius boundary conditions $^{11}$ in the $x$ direction:

$$
\begin{gathered}
\psi\left(x,-L_{y} / 2\right)=\psi\left(x, L_{y} / 2\right)=0 \quad \text { (Dirichlet BC) }, \\
\psi\left(x+L_{x}, y\right)=\psi(x,-y) \quad(\text { Möbius BC). }
\end{gathered}
$$

The quantized wave numbers are $k_{y}=\left(\pi / L_{y}\right) n_{y}$ and $k_{x}=\left(2 \pi / L_{x}\right)\left(\left[\frac{1}{2}\right]_{n_{y}}+n_{x}\right)$, where $n_{y}=1,2, \ldots \quad$ and $n_{x}=0, \pm 1, \pm 2, \ldots$ The notation $[\alpha]_{n}$ represents $\alpha$ for $n$ $=$ even and 0 for $n=$ odd. In the cylinder geometry, Eq. (3) should be replaced by $\psi\left(x+L_{x}, y\right)=\psi(x, y)$, and gives $k_{x}$ $=\left(2 \pi / L_{x}\right) n_{x}$. Thus, only the $n_{y}=$ even eigenstates are affected by the switch from the conventional cylinder (periodic) boundary conditions to the Möbius ones. 
In the absence of disorder, the energies of the eigenstates both in the Möbius and cylinder strips are given by the formula

$$
E_{n_{x} n_{y}}=\epsilon_{x}\left(k_{x}-\frac{2 \pi \phi}{L_{x}}\right)+\epsilon_{y}\left(k_{y}\right)
$$

where $\epsilon_{x}$ and $\epsilon_{y}$ provide the dispersion relation. Equation (4) is rather general for clean systems. To be more specific, let us model the Möbius strip by a tight-binding Hamiltonian. The Möbius strip is constructed from a rectangular lattice including $N \times 2 M$ sites. The rectangle is twisted by $180^{\circ}$, and its two sides are connected, such that longitudinal wire 1 is attached to wire $2 M$, wire 2 is attached to wire $2 M-1$, and so on. The Möbius strip so constructed includes $M$ longitudinal wires with $2 N$ sites on each one. The Hamiltonian is then

$$
\begin{aligned}
H_{\text {Möius }}= & \sum_{n=1}^{2 N} \sum_{m=1}^{M}\left[\varepsilon_{n m} c_{n m}^{\dagger} c_{n m}-t_{1} e^{-2 \pi i \phi / N} c_{n m}^{\dagger} c_{n+1 m}\right] \\
& -t_{2} \sum_{n=1}^{2 N} \sum_{m=1}^{M-1} c_{n m+1}^{\dagger} c_{n m}-\frac{t_{2}}{2} \sum_{n=1}^{2 N} c_{n M}^{\dagger} c_{n+N M}+\text { H.c., }
\end{aligned}
$$

where $c_{n m}$ is the fermion operator at the site $(n, m)$ $(n=1,2, \ldots, 2 N, m=1,2, \ldots, M)$ and $t_{1}$ and $t_{2}$ are longitudinal and transverse hopping amplitudes, respectively. The quantity $\varepsilon_{n m}$ is the site energy. Connecting the two sides of the rectangle without twisting, we obtain a cylindrical strip that includes $2 M$ longitudinal wires composed of $N$ sites. The Hamiltonian of the cylinder is

$$
\begin{aligned}
H_{\text {cylinder }}= & \sum_{n=1}^{N} \sum_{m=1}^{2 M}\left[\varepsilon_{n m} c_{n m}^{\dagger} c_{n m}-t_{1} e^{-2 \pi i \phi / N} c_{n m}^{\dagger} c_{n+1 m}\right] \\
& -t_{2} \sum_{n=1}^{N} \sum_{m=1}^{2 M-1} c_{n m+1}^{\dagger} c_{n m}+\text { H.c. }
\end{aligned}
$$

Locally the two Hamiltonians (5) and (6) look the same. But there are a couple of essential differences between them.

(a) The Möbius Hamiltonian (5) includes an extra term that describes long-range hopping between distant parts of the $M$ th wire. ${ }^{11}$

(b) While the magnetic phase accumulated along the longitudinal direction on each link is the same (that is, $2 \pi \phi / N)$, the corresponding number of links is different ( $2 N$ for the Möbius strip and $N$ for the cylinder).

\section{THE SPECTRUM}

We first consider a system without disorder, namely, $\varepsilon_{n m}=0$. The dispersion relation for an electron in the Möbius strip reads

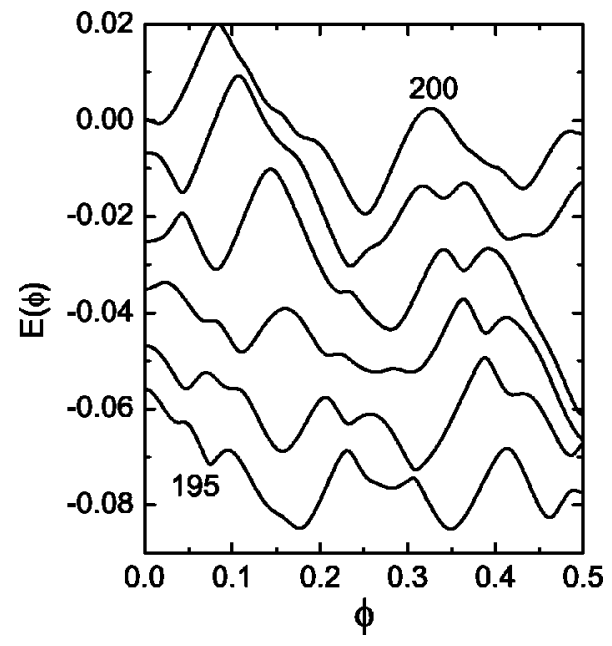

FIG. 1. Single-particle energy spectrum as a function of flux threading the Möbius ring. The 195th-200th energy levels are shown. The parameters are $N=20, M=10, t_{2}=0.5$, and $W=0.5$. Energies are measured in units of $t_{1}$.

$$
\begin{aligned}
E_{n_{x} n_{y}}= & -2 t_{1} \cos \left[\frac{2 \pi}{N}\left(\left[\frac{1}{2}\right]_{n_{y}}+n_{x}-\phi\right)\right] \\
& -2 t_{2} \cos \left(\frac{\pi}{2 M+1} n_{y}\right),
\end{aligned}
$$

where $n_{x}=1, \ldots, N$ and $n_{y}=1, \ldots, 2 M$. Defining new indices $k=[1]_{n_{y}}+2 n_{x}$ and $q=\left[\frac{1}{2}\right]_{k}+n_{y} / 2$, one obtains a more suggestive form,

$$
E_{k q}=-2 t_{1} \cos \left[\frac{\pi}{N}(k-2 \phi)\right]-2 t_{2} \cos \left[\frac{\pi}{2 M+1}\left(2 q-[1]_{k}\right)\right],
$$

where $k=1, \ldots, 2 N$ and $q=1, \ldots, M$. It is instructive to compare it with the energy in the cylinder geometry,

$$
E_{k q}=-2 t_{1} \cos \left[\frac{2 \pi}{N}(k-\phi)\right]-2 t_{2} \cos \left(\frac{\pi}{2 M+1} q\right),
$$

where $k=1, \ldots, N$ and $q=1, \ldots, 2 M$. Despite the apparent similarity between these two spectra, there are at least two important differences. First, the combination of flux and longitudinal momentum is distinct, namely, it is $k-\phi$ for the cylinder and $k-2 \phi$ for the Möbius strip. For a small ratio $t_{2} / t_{1}$ this might affect the periodicity of the current. ${ }^{10} \mathrm{Sec}-$ ond, the miniband structure is different.

We now turn to elucidate the current in disordered Möbius strips. The random numbers $\varepsilon_{n m}$ are assumed to be uniformly distributed over the range $-W / 2 \leqslant \varepsilon_{n m} \leqslant W / 2$, where $W$ represents the strength of disorder. The Hamiltonian Eq. (5) [or Eq. (6)] is treated numerically. As an example, the evolution of single-particle energies with flux in a disordered Möbius strip with $N=20$ and $M=10$ is shown in Fig. 1. The parameters are $t_{2} / t_{1}=0.5$ and $W / t_{1}=0.5$. The pattern of avoided crossing turns out to be remarkably different from that for a cylinder (see Fig. 1 of Ref. 5 therein). It must then be reflected in the behavior of persistent currents. 

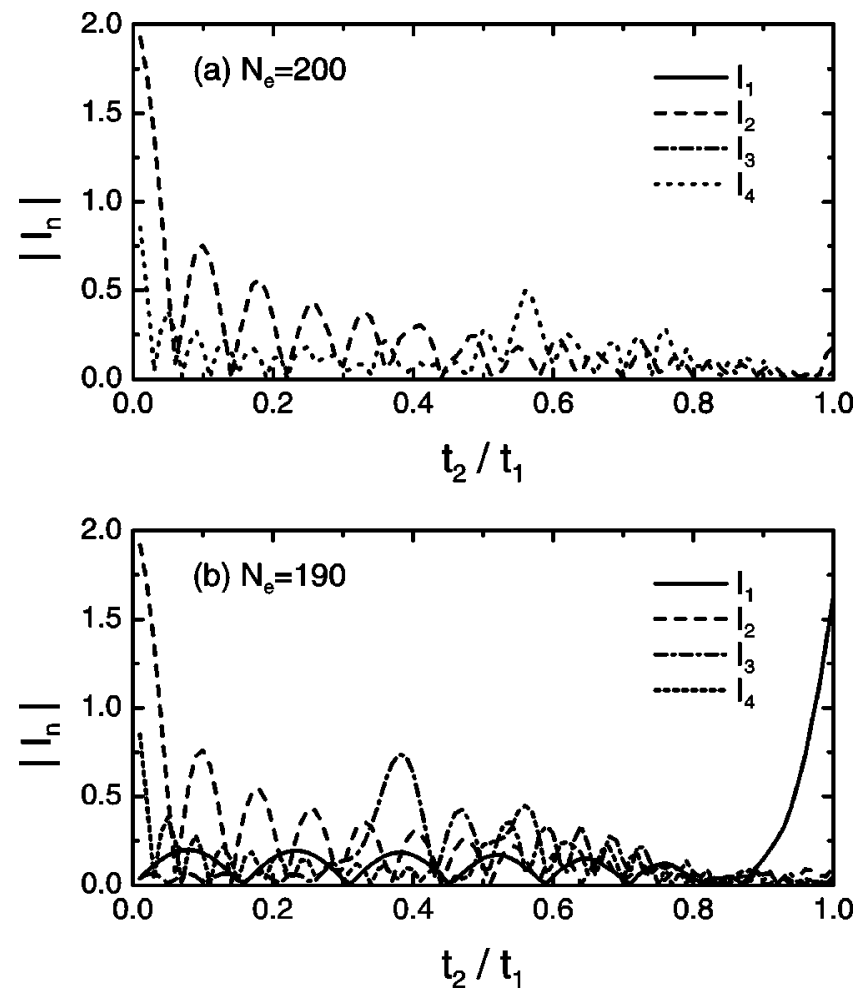

FIG. 2. Fourier components of the persistent current for the clean Möbius strip as a function of the transverse-hopping energy (a) at the half filling and (b) below the half filling. The size of the Möbius strip is given by $N=20$ and $M=10$.

The first stage of the analysis is an inspection of the typical values of $I_{n}$, aiming in determination of their dependence on the ratio $t_{2} / t_{1}$. As expected, in the absence of averaging we find typical $I_{1}$ dominance in case of the cylinder geometry irrespective of the $t_{2} / t_{1}$ ratio. For the Möbius geometry the emerging picture is quite different. Figure 2 shows the Fourier components of the persistent current for a clean Möbius strip as a function of the ratio $t_{2} / t_{1}$ at and below the half filling $\left(N_{e}=200\right)$. For small ratios $\left(t_{2} / t_{1}<0.1\right)$ we find, as can be naively expected, $I_{2}$ dominance. The expected effect of averaging in the cylinder case is to emphasize the $I_{2}$ contribution, while in the Möbius case the expected effect is to emphasize the $I_{4}$ contribution. For clean Möbius strip the $I_{n}$ with odd $n$, as a function of the number of electrons $N_{e}$, is antisymmetric around half filling. Therefore $I_{1}$ and $I_{3}$ completely vanish [Fig. 2(a)] (see further discussion in Sec. V). To avoid this particularity at the half filling, we display in Fig. 2(b) also the case where the number of electrons $\left(N_{e}\right.$ $=190)$ is below half filling. For large ratios $\left(t_{2} / t_{1}>0.8\right)$ we observe in Fig. 2(b) a cylinderlike regime where there is typically $I_{1}$ dominance. This is because the strong transverse hopping changes the periodicity of the Möbius strip to that of the conventional cylinder. The somewhat unexpected observation is that there is a distinct wide intermediate regime $\left(0.1<t_{2} / t_{1}<0.8\right)$ where $I_{1}, I_{2}, I_{3}$, and $I_{4}$ are all comparable. This is the regime that is of experimental relevance. The expected effect of averaging in this regime is to emphasize both the $I_{2}$ and the $I_{4}$ contributions.

\section{STATISTICAL ANALYSIS}

The problem arising in the analysis of persistent currents in disordered Möbius strips is how to characterize the statistics of the calculated data. It was already pointed out that essential properties of observables result from the averaging procedure and the nature of the underlying statistical ensemble. ${ }^{4-6}$ On the other hand, fabrication of a Möbius strip requires an outstanding effort, ${ }^{7}$ and hence, anticipated measurements of the persistent current would probably be performed on a single sample. Thus, somewhat unfortunately, the important results reported therein and the powerful calculation methods based on supersymmetry might be less useful for single-sample experiments since there is no averaging.

What is then the most efficient way to present our calculated results? The answer is provided by elementary statistics. An experimental result consists of a set of $K$ measurements $I\left(\phi_{i}\right), i=1,2, \ldots, K$ performed on a given sample. This sample is taken out of an ensemble of Möbius strips with different disorder realizations, electron numbers $N_{e}$, aspect ratios, etc. The set $\left\{I\left(\phi_{i}\right) \mid i=1, \ldots, K\right\}$ can be regarded as an instance of a random vector in a $K$ dimensional space. Alternatively, this instance can be represented by the current harmonics $\left(I_{1}, I_{2}, \ldots\right)$ defined via Eq. (1). For our purpose it seems adequate to keep only the first four harmonics. The relevant statistical ensemble is then a set of "points" $\left(I_{1}, I_{2}, I_{3}, I_{4}\right)$ in four-dimensional probability space, each point corresponds to a possible experimental measurement of the current on the entire $\phi$ interval. Let us denote the number of points within an infinitesimal four-dimensional volume element by $P\left(I_{1}, I_{2}, I_{3}, I_{4}\right) d I_{1} d I_{2} d I_{3} d I_{4}$. The distribution function $P$ is normalized to $\mathcal{N}$, the total number of members in the ensemble. The most probable (typical) experimental result is then determined by the quadruple $I_{1}, I_{2}, I_{3}, I_{4}$ at which $P$ is maximal. Another quantity, which seems more informative and easy to analyze, is the distribution

$$
p_{n}\left(I_{n}\right)=\int_{0}^{\left|I_{n}\right| / 2} P\left(I_{1}, I_{2}, I_{3}, I_{4}\right) \prod_{m \neq n} d\left|I_{m}\right|
$$

This corresponds to the possibility of finding a sample whose current $I(\phi)$ is approximately described by $I(\phi)$ $\approx I_{n} \sin (2 \pi n \phi)$. [For a sample counted by $p_{n}\left(I_{n}\right)$, all the harmonics other than $I_{n}$ are at most half of $I_{n}$ in magnitude]. The number of members in the ensemble that exhibit $I_{n}$ dominance is therefore $\mathcal{N}_{n}=\int_{0}^{\infty} p_{n}\left(I_{n}\right) d\left|I_{n}\right|$. If $\mathcal{N}_{n}>\mathcal{N}_{m}$ for any $m \neq n$, the typical periodicity of $I(\phi)$ is dominantly $1 / n$.

In actual calculations, we assume that the lattice structure, the aspect ratio, and the strength of disorder are fixed, and that the temperature is very low. Then, two quantities are still fluctuating, namely, the filling factor (or the electron number $N_{e}$ ) and the specific realization of disorder. We generate an ensemble of $\mathcal{N}=\mathcal{N}^{a} \mathcal{N}^{b}$ members corresponding to $\mathcal{N}^{a}$ consecutive values of $N_{e}$, usually around half filling, and $\mathcal{N}^{b}$ realizations of disorder for each one of them. Actually, for our systems of size $N=20, M=10$ with $t_{1}=1, t_{2}=0.5$, and $W=0.5$, we take $150 \leqslant N_{e} \leqslant 250$, hence $\mathcal{N}^{a}=101$ and $\mathcal{N}^{b}$ 


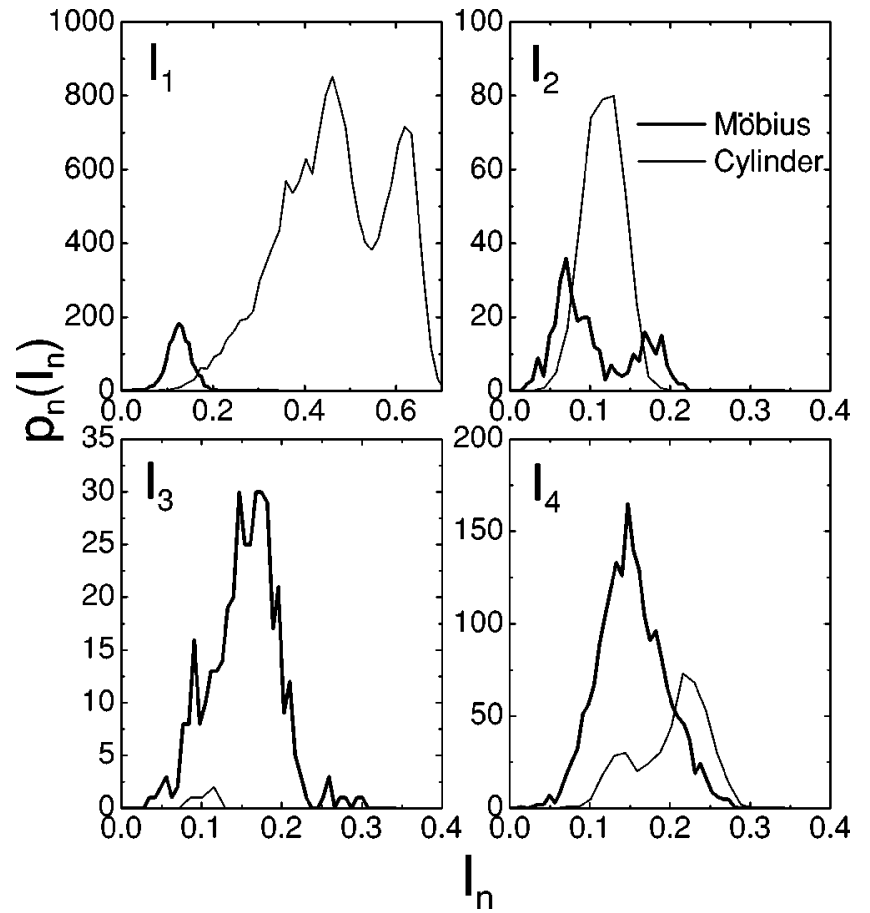

FIG. 3. The distributions $p_{n}\left(I_{n}\right)(n=1,2,3,4)$ defined by Eq. (9) for the cylinder and Möbius ensembles. The numbers of members with $I_{n}$ dominance are $\mathcal{N}_{1}=15,829, \mathcal{N}_{2}=382, \mathcal{N}_{3}=4$, and $\mathcal{N}_{4}$ $=439$ for the cylinder ensemble, and $\mathcal{N}_{1}=1,562, \mathcal{N}_{2}=336, \mathcal{N}_{3}$ $=384$, and $\mathcal{N}_{4}=1,992$ for the Möbius ensemble.

$=250$, so that $\mathcal{N}=25250$. The distributions $p_{n}\left(I_{n}\right)$ for the cylinder and Möbius ensembles are shown in Fig. 3.

\section{MAIN OBSERVATIONS}

The most striking result that can be deduced from Fig. 3 is the essential reduction of $\mathcal{N}_{1}$ for the Möbius ensemble compared with the cylinder one. For the present ratio $N / 2 M$ $=1$, there is also a strong tendency towards $\Phi_{0} / 4$ periodicity, since $\mathcal{N}_{4}>\mathcal{N}_{m \neq 4}$ for the Möbius ensemble. This result is intriguing, because here we have no averaging procedure that is crucial to get the $1 / 2$ periodicity in cylindrical strips. However, this $1 / 4$ periodicity emerges only for the specific ratio $N / 2 M=1$. We have calculated the distributions $p_{n}\left(I_{n}\right)$ for Möbius strips with several aspect ratios. The value of $\mathcal{N}_{n}$ depends on the aspect ratio. No specific $n$ gives prominent $\mathcal{N}_{n}$ independently of the aspect ratio. On the other hand, the collapse of $I_{1}$ dominance in the Möbius ensemble is robust and persists in systems with different ratios $N / 2 M$ as well. We can safely say that $\mathcal{N}_{1}, \mathcal{N}_{2}, \mathcal{N}_{3}$, and $\mathcal{N}_{4}$ become all comparable in the Möbius ensemble.

The natural question that comes to mind is whether this result is a consequence of the Möbius geometry or, rather, is it due to the presence of disorder. In order to answer this question, we have performed the calculation of $P^{0}\left(I_{1}, I_{2}, I_{3}, I_{4}\right)$ for a "clean" Möbius ensemble (without disorder, only $N_{e}$ is being changed). We found that the probability to find any $I_{n}$ dominance is extremely small. The immediate conclusion is that disorder is essential for the
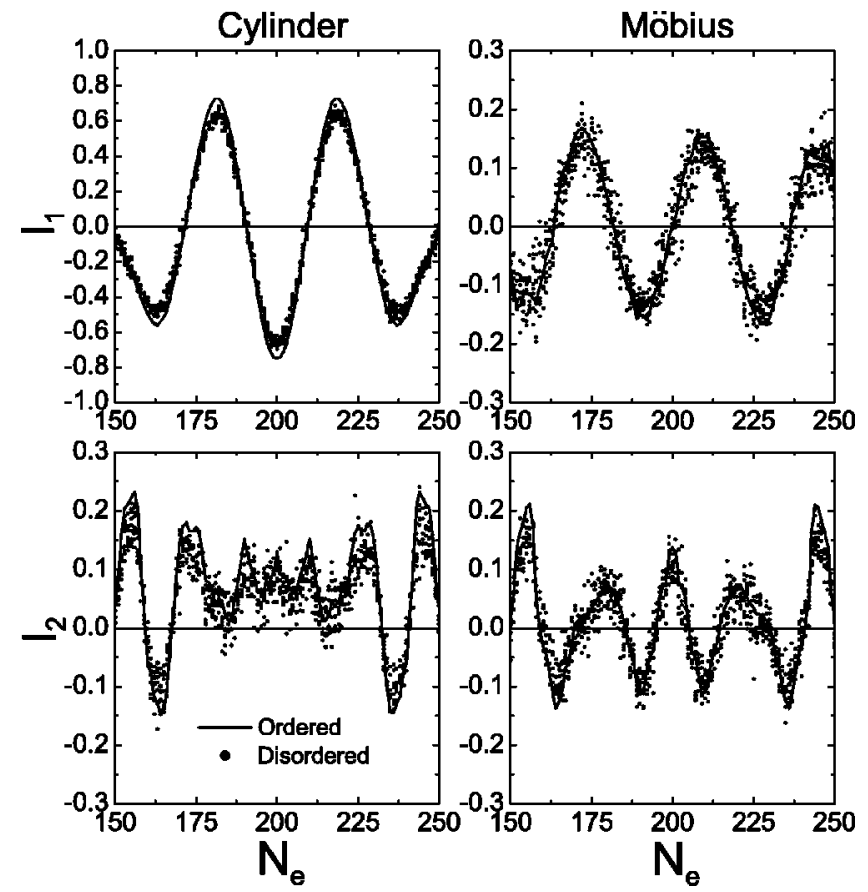

FIG. 4. $I_{1}$ and $I_{2}$ as functions of $N_{e}$ for the ordered (solid line) and disordered (dots) systems. Parameters describing the systems are the same as those for Fig. 3.

identification of Möbius strips via $I_{n>1}$ dominance. Does this mean that interference or weak-localization effects due to the presence of disorder are important? To clarify this point, we should understand how $P^{0}\left(I_{1}, I_{2}, I_{3}, I_{4}\right)$ is modified by disorder. The distribution $P^{0}\left(I_{1}, I_{2}, I_{3}, I_{4}\right)$ is, in fact, a function defined on a one-dimensional curve $\left[I_{1}\left(N_{e}\right), I_{2}\left(N_{e}\right), I_{3}\left(N_{e}\right), I_{4}\left(N_{e}\right)\right]$ in $\left(I_{1}, I_{2}, I_{3}, I_{4}\right)$ space. For this reason, it is unlikely to find a sample where one of the $I_{n}$ is dominant. The effect of disorder is to give some "thickness" to this curve (see Fig. 4). Taking into account that the amplitudes of $I_{n}\left(N_{e}\right)$ for Möbius strips are all comparable, the thickness gives a finite probability to find samples where one of the $I_{n}$ is dominant. On the contrary, in the case of cylindrical strips, the amplitude of $I_{1}\left(N_{e}\right)$ is overwhelmingly larger than those of $I_{n \neq 1}\left(N_{e}\right)$, which makes it unlikely to find $I_{n \neq 1}\left(N_{e}\right)$ dominated samples even if we take the statistical effect of disorder into account. We should note here that the function $I_{n}$ with odd $n$ for the clean Möbius strip is an even function around half filling $\left(N_{e}=200\right)$ and an odd function for odd $n$, while the function $I_{n}$ for arbitrary $n$ is an even function in the cylinder case.

Our findings regarding $\mathcal{N}_{n}$ for the Möbius ensemble are based on the fact that the amplitudes of $I_{n}\left(N_{e}\right)$ are all comparable for Möbius strips. As we have observed in Fig. 2, this is a robust statistical property in the intermediate regime $0.1<t_{2} / t_{1}<0.8$. The choice $t_{2} / t_{1}=0.5$ above provides typical results for $p_{n}\left(I_{n}\right)$ and $\mathcal{N}_{n}$ in case that $t_{2} / t_{1}$ is within this distinct regime.

\section{CONCLUSIONS}

We have studied the persistent currents of noninteracting electrons in Möbius strips. The spectral properties for a clean 
system were found analytically, and the effect of disorder on the currents was analyzed numerically. We have found that disorder is quite essential for the identification of Möbius strips. The issue of disorder averaging is not relevant for single sample experiments, and hence, special care is required for statistical analysis of the current harmonics. The fingerprint of the Möbius geometry is an enhanced probability to find samples in which $I_{n}$, with $n>1$, dominates. This should be contrasted with the case of cylinder geometry, where there is a clear $I_{1}$ dominance. The above assertion regarding the fingerprint of the Möbius geometry is correct, provided the effect of disorder is properly taken into account.

\section{ACKNOWLEDGMENTS}

We would like to thank T. Nakayama for very helpful discussions. One of the authors (Y.A.) was supported by the Invitation Fellowship for Research in Japan (Short Term) of the Japan Society for the Promotion of Science. Numerical calculations in this work have been mainly performed on the facilities of the Supercomputer Center, Institute for Solid State Physics, University of Tokyo.
${ }^{1}$ M. Büttiker, Y. Imry, and R. Landauer, Phys. Lett. 96A, 365 (1983).

${ }^{2}$ B.L. Altshuler, A.G. Aronov, B.Z. Spivak, D.Yu. Sharvin, and Yu.V. Sharvin, Pis'ma Zh. Eksp. Teor. Fiz. 35, 484 (1982) [JETP Lett. 35, 588 (1982)].

${ }^{3}$ L. Lévy, G. Dolan, J. Dunsmir, and H. Bouchiat, Phys. Rev. Lett. 64, 2074 (1990).

${ }^{4}$ H.F. Cheung, E.K. Riedel, and Y. Gefen, Phys. Rev. Lett. 62, 587 (1989).

${ }^{5}$ Hélène Bouchiat and Gilles Montambaux, J. Phys. (France) 50, 2695 (1989).

${ }^{6}$ B.L. Altshuler, Y. Gefen, and Y. Imry, Phys. Rev. Lett. 66, 88 (1991).

${ }^{7}$ S. Tanda, T. Tsuneta, Y. Okajima, K. Inagaki, K. Yamaya, and N. Hatakenaka, Nature (London) 417, 397 (2002).

${ }^{8}$ V. Chandrasekhar, R.A. Webb, M.J. Brady, M.B. Ketchen, W.J.
Galager, and A. Kleinsasser, Phys. Rev. Lett. 67, 3578 (1991).

${ }^{9}$ It has been theoretically predicted that a novel state appears in a superconducting Möbius strip under a magnetic field: M. Hayashi, and H. Ebisawa, J. Phys. Soc. Jpn. 70, 3495 (2001).

${ }^{10}$ Fredric Mila, C.A. Stafford, and Sylvian Capponi, Phys. Rev. B 57, 1457 (1998).

${ }^{11}$ If, instead of Eq. (2), we assume the periodic BC in the $y$ direction, the Möbius BC of Eq. (3) are modified as $\psi\left(x+L_{x}, y\right)$ $=\psi\left(x, y+L_{y}\right)$. This leads to a long-range hopping term in Eq. (5), which does not "discriminate" the $M$ th wire. Yet another possibility is to keep Eq. (3). This possibility is of academic interest, since it corresponds to a geometry that cannot be embedded in a three-dimensional Euclidean space. In the latter case, there is a symmetry breaking that prevents having a stationary current in the $y$ direction. 